\title{
Growth of a dense Gadolinium metal-organic framework on oxide-free Silicon for cryogenic local refrigeration
}

\author{
Giulia Lorusso, ${ }^{a}$ Eva Natividad, ${ }^{a}$ Marco Evangelisti ${ }^{a}$ and Olivier Roubeau ${ }^{\text {*a }}$ \\ ${ }^{a}$ Instituto de Ciencia de Materiales de Aragón (ICMA), CSIC and Universidad de Zaragoza, Plaza San Francisco s/n, 50009 Zaragoza, Spain
}

Certain magnetic molecules and molecular-based frameworks are considered as alternative materials for cryogenic cooling through their large magneto-caloric effect. This potential is particularly appealing for applications requiring local refrigeration, but so far no study has been aimed at making thin films of efficient molecular coolers on typical device substrate and evaluate their cooling power. In this work, the growth of a dense gadolinium metal-organic framework on oxide-free silicon covered by either self-assembled or covalent monolayers with carboxylic end groups is explored. A continuous coverage with nanosized crystallites of the selected gadolinium formate framework is successfully formed on covalent monolayers obtained by hydrosilylation with either undecenoic acid or undecynoic acid. The thickness of the magnetocaloric deposit can be increased by increasing growth time, as shown by the surface densities derived through determination of the film magnetic properties. An estimation of the cooling potential of the thicker film obtained shows that it would refrigerate a $2 \mu \mathrm{m}$ thick Si membrane from 5 to $0.8 \mathrm{~K}$, by adiabatic demagnetization from $2 \mathrm{~T}$ to zero field. Altogether, this study demonstrates the potential of molecular coolers thin films to develop on-chip magnetic refrigeration at cryogenic temperatures.

\section{Introduction}

Molecular nanomagnets are a class of materials based on isolated coordination complexes composed of one to numerous interacting paramagnetic metal ions. Their peculiar magnetic properties at the molecular scale have resulted in their proposed use in various applications including (quantum) information storage and processing, ${ }^{1}$ spin-based quantum computing $^{2}$ and cryogenic refrigeration. ${ }^{3}$ The latter relies on materials with a large magneto-caloric effect (MCE), ${ }^{4}$ i.e., important changes of the magnetic entropy $\Delta S_{\mathrm{m}}$ and adiabatic temperature $\Delta T_{\text {ad }}$ associated with a change in the applied magnetic field $\Delta B$. Adiabatic demagnetization refrigerators typically use oxides, such as gadolinium gallium garnet (GGG), and inorganic paramagnetic salts, such as cerium magnesium nitrate (CMN) or chromic potassium alum (CPA), to allow cooling to respectively liquid-helium temperatures and ultra-low temperatures, being valid alternatives to the use of liquid-helium itself or to ${ }^{3} \mathrm{He}-{ }^{4} \mathrm{He}$ dilution fridges. ${ }^{5}$ Although the currently used materials are well suited and developed, ${ }^{6}$ molecular-based materials have been proposed as alternatives on basis of the possibilities offered by molecularscale rational synthetic design. ${ }^{3}$ Indeed, minimizing anisotropy and maximizing the density of spin carrier, while avoiding

\section{Conceptual insights}

We present a method to provide solid-state devices with insitu cooling potential for low temperatures, through the formation of a thin layer of an adequate metal organic framework material. Gadolinium molecular-based materials have arisen as alternative coolants in magnetic refrigeration applications, which rely on the magnetocaloric effect of paramagnetic solids. This is particularly true for situations in which their relatively low thermal conductivity is not detrimental, i.e. for application requiring local cooling. Surprisingly, there has been no study aimed as yet at making thin films of efficient molecular magnetic coolers on typical substrates used in electronic device elaboration. The strategy used in this work allows the formation on oxide-free silicon of a continuous layer of crystallites of gadolinium formate, one of the best molecular-based cooler due to its high relative density of magnetic elements. Estimation of the cooling power of the deposit demonstrate that silicon membranes would be efficiently cooled from $5 \mathrm{~K}$ to sub-Kelvin temperatures by a single adiabatic demagnetization, surpassing the electronic schemes currently used for such local cooling, and therefore validating the proposed approach.

long-range magnetic order, has brought the MCE of molecular-based systems to values competing with currentlyused materials. ${ }^{7,8}$ Unfortunately, their use in bulk applications might be severely hampered by the low thermal conductivity that can be expected from their molecular nature, an aspect that has remained somewhat underscored while these socalled molecular coolers were arousing increased interest. ${ }^{9}$ We have recently reported the first evaluation of the cryogenic thermal conductivity of an archetypal molecular cooler, $\left[\mathrm{Gd}_{2}(\mathrm{OAc})_{6}\left(\mathrm{H}_{2} \mathrm{O}\right)_{4}\right] \cdot 4 \mathrm{H}_{2} \mathrm{O}$, confirming this important limitation. ${ }^{10}$ We also have evaluated the relative improvement that can be obtained through the elaboration of composite materials with a component with a good thermal conductivity, ${ }^{10}$ although the gain remained limited. Nevertheless, direct measurements of the MCE of samples of different thicknesses have also shown that thinner samples or layers are not affected by adverse effects of the material poor thermal conductivity. ${ }^{10}$ Basically, a sufficiently reduced thickness makes the temperature gradients within the material in contact with a thermal bath, a consequence of its poor thermal conductivity, negligible. This in turns minimizes any related loss of cooling power. A low thermal conductivity may therefore not represent such a stringent limitation if the molecular cooler is to refrigerate locally, for example, as coating on a membrane. In such conditions, even if the thermal conductance at the interface will still play a role, the molecular cooler should be able to efficiently cool the material it is in contact with. Here, 


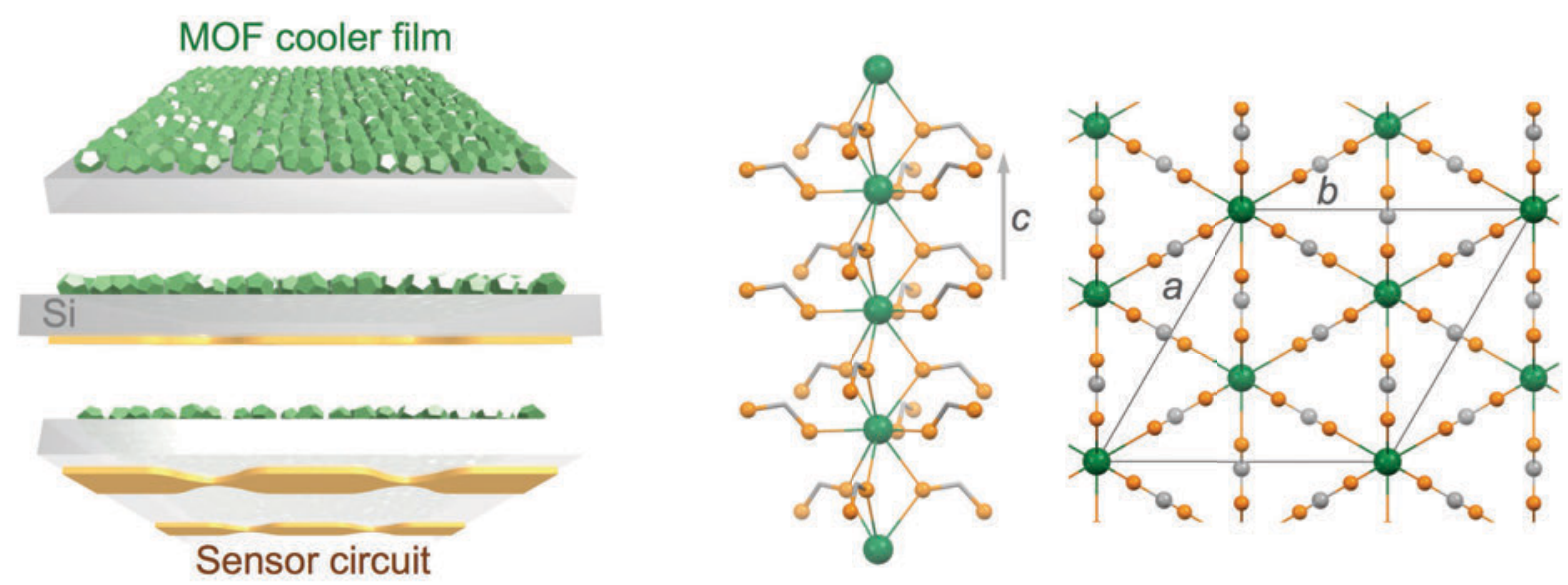

Scheme 1. Left: schematic representation of an integrated device in which a superficial layer of molecular-based cooler particles would allow local microrefrigeration of a silicon membrane and a sensor circuit built on its surface. Right: views of the structure of the gadolinium formate $\mathrm{MOF}$ Gd(HCOO) 3 , selected here for its high magneto-caloric effect. Colour code: Gd, green spheres; O, orange spheres; C, grey spheres or sticks.

materials possess an advantage in that they may be processed in solution, and therefore assembled and integrated directly on the surface to be cooled.Such ability has been demonstrated, and the large MCE of the molecular materials shown to be maintained in their deposits. ${ }^{11,12} \mathrm{~A}$ strategy using membranes coated with molecular coolers as local microrefrigerators would have a significant interest in any application requiring local, fast and light refrigeration, for example, in sensors used in space borne missions (Scheme 1 left). ${ }^{13}$ For such objective, the choice of the substrate is imposed by the necessity of a low heat capacity, combined with easy manipulation and good thermal conductivity. Among typical substrates used in device fabrications, Si is clearly the one with the lowest heat capacity in the temperature range of application, typically below $10 \mathrm{~K}$, with a Debye temperature of $645 \mathrm{~K}$ vs. $170 \mathrm{~K}$ for Au. Then, the molecular-based cooler material should have the largest volumetric MCE, and in this respect dense $\mathrm{Gd}$ (III) metal-organic frameworks (MOFs) appear as ideal. Here, we have selected the gadolinium formate MOF, $\mathrm{Gd}(\mathrm{HCOO})_{3}$, which has a dense 3D framework hexagonal structure made of $\mathrm{Gd}(\mathrm{III})$ chains connected through the formate carboxylic groups (Scheme 1 right), making it one of the molecular-based coolers with the highest volumetric MCE. ${ }^{8 a}$ Covering a metallic surface with a MOF material involves its pre-functionalization, a method of choice being the formation of self-assembled monolayers (SAMs) with adequate end chemical functions, in the present case carboxylic acid groups. There is now abundant literature on the growth of MOFs on a variety of substrates, ${ }^{14}$ but the focus has merely been on a limited number of porous MOFs. SAMs can be formed on the native oxide layer of Si substrates, but good quality SAMs normally require the formation of a better oxide layer by etching the native oxide layer and oxidation/plasma. This oxide layer is highly detrimental for cooling due to its poor thermal conductivity, ${ }^{15}$ and it is therefore preferable to make SAMs on oxide-free Si. This work therefore explores the growth of $\mathrm{Gd}(\mathrm{HCOO})_{3}$ on oxide-free $\mathrm{Si}$ substrates functionalized with carboxylic acid groups monolayers, either physisorbed using thiol-based SAMs or covalent through hydrosilylation reactions. The cooling power of the resulting $\mathrm{Gd}(\mathrm{HCOO})_{3}$ thin films is evaluated through determination of their low-temperature magnetic properties.

\section{Experimental}

\subsection{Materials synthesis.}

All reagents were purchased from Aldrich and used as received. HF (48\% in water), ethylacetate $(>99 \%, \mathrm{GC}), 1,2-$ dimethoxyethane $(>99 \%$, reagent grade) and acetone (>99.5\%, reagent grade) were purchased from Aldrich, while $\mathrm{CH}_{2} \mathrm{Cl}_{2}(99.8 \%+50 \mathrm{ppm}$ amylene, synthesis grade) and absolute $\mathrm{EtOH}$ (99.9\%, synthesis grade) were obtained from Scharlab. The water used in all manipulation was Milli-Q water (resistivity > $18 \mathrm{MWcm}$ ). $\mathrm{Si}(100)$ and $\mathrm{Si}(111)$ wafers were purchased from Si-Mat. Au-covered Si substrates were obtained by evaporating $10 \mathrm{~nm}$ of $\mathrm{Cr}$, to improve adhesion, and $100 \mathrm{~nm}$ of Au onto a 4 inches Si(100) wafer. Pieces of variable sizes in the range $0.25-2 \mathrm{~cm}^{2}$ were cut out of the different types of substrates.

Synthesis of $\mathrm{Gd}(\mathrm{HCOO})_{3} \cdot \mathrm{GdCl}_{3} \cdot 6 \mathrm{H}_{2} \mathrm{O}(99 \%, 3.66 \mathrm{~g}, 9.75$ $\mathrm{mmol}$ ) dissolved in $100 \mathrm{~mL} \mathrm{EtOH}$ and ammonium formate $(97 \%, 1.93 \mathrm{~g}, 29.69 \mathrm{mmol})$ dissolved in $100 \mathrm{~mL} \mathrm{EtOH}$ were mixed and stirred at $60^{\circ} \mathrm{C}$ for 3 hours. After cooling to RT, the fine white precipitate was recovered by filtration on a membrane (0.45 $\mu \mathrm{m}$, Durapore), washed with little EtOH and dried. The yield of bulk $\mathrm{Gd}(\mathrm{HCOO})_{3}$ powder was $2.71 \mathrm{~g}(9.27$ $\mathrm{mmol}, 95 \%$ based on $\mathrm{Gd}$ ). Phase purity was checked through IR spectroscopy and powder XRD (Figure S1). The solubility of $\mathrm{Gd}(\mathrm{HCOO})_{3}$ in $\mathrm{EtOH}$ was determined to be of the order of $1.1 \mathrm{mmolL}^{-1}$, and solutions slightly below saturation were used for growth on the different Si substrates. 
Self-Assembled Monolayers on $\mathrm{Si}(100)$ and $\mathrm{Au} / \mathrm{Cr} / \mathrm{Si}(100)$ substrates. After bath sonication in acetone to remove organic impurities and drying with $\mathrm{N}_{2}$, the substrates were dipped into a $2 \%$ aqueous $\mathrm{HF}$ for $2 \mathrm{~min}$, rinsed with Milli-Q water and dried with $\mathrm{N}_{2}$. Once dried, they were immediately soaked into an EtOH solution of the selected thiol, either 1undecanethiol (UDT, 98\%), 11-mercapto-undecanoic acid (11MUDA, 95\%) or 16-mercapto-hexadecanoic acid (16-MHDA, $90 \%$ ), and maintained immersed for $24 \mathrm{~h}$. The substrates were rinsed thoroughly with $\mathrm{EtOH}$ and used right away. The concentration of the thiol was $5 \mathrm{mmol}$ for $\mathrm{Si}(100)$ substrates and $1 \mathrm{mmol}$ for $\mathrm{Au} / \mathrm{Cr} / \mathrm{Si}(100)$ substrates.

Monolayers by thermal hydrosilylation of $\mathrm{H}-\mathrm{Si}(111)$ with alkenes. First, hydrogen-terminated $\mathrm{Si}(111)$ surfaces were prepared. Each cut piece of $\mathrm{Si}(111)$ was first washed successively under a flow of Milli- $Q$ water, ethylacetate and acetone, before being subjected to $5 \mathrm{~min}$ bath sonication in acetone, rinsed with Milli-Q water and dried with a stream of $\mathrm{N}_{2}$. The substrates were then immersed in $2 \%$ aqueous HF for 2 min and finally etched in a $40 \%$ aqueous $\mathrm{NH}_{4} \mathrm{~F}$ solution for 3 to $15 \mathrm{~min}$, after which they were thoroughly rinsed with Milli-Q water, dried with a stream of $\mathrm{Ar}$ and used right away.

The dried substrates were immediately transferred to a roundbottom flask under $\mathrm{Ar}$ containing the alkene, either 1undecene $(97 \%)$ or 10 -undecenoic acid $(98 \%)$, in sufficient amount to cover the $\mathrm{H}-\mathrm{Si}(111)$ substrates. Under a continuous $\mathrm{Ar}$ flow, the temperature was then raised to $175^{\circ} \mathrm{C}$ in about 20 min and kept for $4 \mathrm{~h}$ at $178-182^{\circ} \mathrm{C}$. After cooling to ca. $50^{\circ} \mathrm{C}$, the alkene was removed and the substrates washed twice with ethylacetate and twice with $\mathrm{CH}_{2} \mathrm{Cl}_{2}$ to remove the excess unreacted alkene. After a final washing with acetone, the substrates were dried under a flow of $\mathrm{N}_{2}$ and used right away.

Monolayers by light-activated hydrosilylation of $\mathrm{H}-\mathrm{Si}(111)$ with alkynes. The neat alkyne in sufficient amount to cover the substrate(s) was charged in a small three-neck flask with an $\mathrm{Ar}$ inlet and reflux condenser connected to vacuum pump. The alkyne, either 1-dodecyne (98\%) or 10-undecynoic acid $(95 \%)$, was first melted by warming to ca. $60^{\circ} \mathrm{C}$ under Ar flow, and the temperature was then maintained constant. The pressure in the flask was then reduced to ca. 10 mbar and the alkyne deoxygenated with Ar for $30 \mathrm{~min}$. After filling with Ar to atmospheric pressure, the fresh $\mathrm{H}-\mathrm{Si}(111)$ substrates were immediately transferred into the alkyne under Ar flow. The flask was closed again, the pressure reduced and the system let under the dynamic flow of $\mathrm{Ar}$ and light from the standard fluorescent tubes from the hood at $60^{\circ} \mathrm{C}$ for at least $15 \mathrm{~h}$. The reaction was stopped by backfilling the flask with $\mathrm{Ar}$, and the alkyne was removed, still at $60^{\circ} \mathrm{C}$. The substrates were then washed with dimethoxyether, $\mathrm{EtOH}$ and $\mathrm{CH}_{2} \mathrm{Cl}_{2}$, twice each, and subjected to sonication for $5 \mathrm{~min}$ in $\mathrm{CH}_{2} \mathrm{Cl}_{2}$ to remove physisorbed molecules, dried with $\mathrm{N}_{2}$ and used right away.

Growth of Gadolinium formate. The freshly prepared substrates with carboxylate-terminated monolayers were immersed into an ethanol solution of $\mathrm{Gd}(\mathrm{HCOO})_{3}$ just below saturation, at ca. $1.0 \mathrm{mmol} \mathrm{L}^{-1}$. The flask was covered to avoid evaporation and contamination and left unperturbed for $24 \mathrm{~h}$. The substrates were then washed with ethanol and dried with a flow of $\mathrm{N}_{2}$. Longer immersions (48h or 2 times $24 \mathrm{~h}$ ) as well as repeated short $(15 \mathrm{~min}$ ) immersions were also tested, the substrate being washed by immersion in pure ethanol in between successive immersions.

\subsection{Physical characterization}

Surface Characterization. Static water contact angles were first determined using a home-made set-up with an estimated accuracy of $\pm 3^{\circ}$ (Milli-Q water, 3 droplets per substrate). Measurements were repeated for some of the substrates using an Attension Theta Lite contact angle meter, with an estimated accuracy of $1^{\circ}$. X-ray Photoelectron Spectroscopy measurements were performed in a Kratos AXIS SUPRA spectrometer, using a monochromatized Al Ka source (1486.6 $\mathrm{eV}$ ) at the Laboratorio de Microscopías Avanzadas (LMA). Wide scans were acquired at analyzer pass energy of $160 \mathrm{eV}$, whereas high-resolution narrow scans were performed at constant pass energy of $20 \mathrm{eV}$. The spectra were obtained at room temperature. The binding energy (BE) scale was internally referenced to the $C 1$ s peak ( $B E$ for $C C=284.9 \mathrm{eV}$ ). Atomic Force Microscopy was done using a NT-MDT Ntegra Aura setup under ambient conditions and in semi-contact mode. Field-Emission Scanning Electron Microscopy (FESEM) images were obtained through the Servicio de Apoyo a la Investigación-SAI, Universidad de Zaragoza, on a Carl Zeiss MERLIN microscope provided with an Energy Dispersive Spectroscopy (EDS) detector INCA 350 from Oxford Instruments. High resolution topographical images were obtained using the in-lens detector of secondary electrons. The samples were coated with a few $\mathrm{nm}$ of carbon.

X-ray Diffraction. Data were obtained at ambient temperature using a Rigaku D-Max diffractometer equipped with a rotating anode and a graphite monochromator to select the CuKa wavelength, through the Servicio General de Apoyo a la Investigación-SAI, Universidad de Zaragoza. Grazingincidence experiments were also performed with a PANalytical Empyrean instrument with a CuKa X-ray tube and PIXcel 1D detector, housed at the Instituto de Nanocienca de Aragón, Universidad de Zaragoza. The data were acquired using parallel beam geometry and an angle of incidence of $0.65^{\circ}$.

Magnetization measurements. Variable-temperature (2 $100 \mathrm{~K}$, at $0.5 \mathrm{~T}$ applied $d c$ field) and variable-field $(0-5 \mathrm{~T}$, at 2 and $5 \mathrm{~K}$ ) magnetization data were obtained with a Quantum Design MPMS-XL through the Servicio de Apoyo a la Investigación-SAI, Universidad de Zaragoza. Experiments were carried out on pieces of Si substrates cut to fit and be held vertically within the standard plastic straws. Areas of the pieces of wafer measured were in the range $0.25-0.65 \mathrm{~cm}^{2}$. For each sample with $\mathrm{Gd}(\mathrm{HCOO})_{3}$ thin film, measurements were done on the corresponding free substrate under the exact same conditions. 


\section{Results and Discussion}

\subsection{Pre-functionalization by monolayer formation and} characterization.

Because basically no studies of MOF growth on oxide-free $\mathrm{Si}$ are available and the optimal growth conditions for a specific MOF are difficult to predict, several approaches have been used here for the formation of carboxylic acid monolayers on oxide-free Si. A first approach involves the formation of SAMs on bare $\mathrm{Si}(100)$ surface with mercapto-undecanoic acid (MUDA) and mercapto-hexadecanoic acid (MHDA) in ethanol, the resulting samples being hereafter denoted $\mathbf{1}\left(\mathbf{C}_{11}\right)$ and $\mathbf{1}\left(C_{16}\right)$. For comparison, the same MUDA SAM was formed on a gold layer grown on $\mathrm{Si}(100)$, denoted $\mathbf{1}^{\mathrm{Au}}\left(\mathbf{C}_{11}\right)$. A second strategy uses the thermally-induced hydrosilylation of hydrogen-terminated Si surface ${ }^{16}$ using neat undecenoic acid, the resulting sample being denoted 2 . The Si(111) surface is preferred in this case due to the ability to form a higher density of $\mathrm{Si}-\mathrm{H}$ bonds. A third strategy involves the light-activated hydrosilylation of $\mathrm{H}-\mathrm{Si}(111)^{17}$ with neat undecynoic acid, the resulting sample being denoted 3 . The same conditions were used to form samples with similar albeit hydrophobic SAMs, i.e., using undecanethiol (denoted $\mathbf{1}\left(\mathrm{C}_{11}\right)^{\text {ref }}$ and $\left.\mathbf{1}^{\mathrm{Au}}\left(\mathrm{C}_{11}\right)^{\mathrm{ref}}\right)$, undecene $\left(2^{\text {ref }}\right)$ and dodecyne $\left(3^{\text {ref }}\right)$, for comparison with literature. These approaches and corresponding abbreviations used are summarized in Table 1.

Static contact angles $\theta_{\mathrm{s}}$ were measured for all monolayers with Milli-Q water drops (Figures S2-S3), and are gathered in Table 1. As expected, monolayers made with alkyl end groups have $\theta_{\mathrm{s}}$ characteristic of hydrophobic surfaces, while those for carboxylic end groups are indicative of hydrophilic surfaces. In particular, the $\theta_{\mathrm{s}}$ of the hydrophobic monolayers obtained by hydrosilylation, $96^{\circ}$ for $2^{\text {ref }}$ and $98^{\circ}$ for $3^{\text {ref }}$, are comparable with those previously reported using an hexadecyl chain. ${ }^{18}$ The corresponding monolayers with carboxylic acid end groups 2 and 3 have similar $\theta_{\mathrm{s}}$, also comparable with that for $1^{\mathrm{Au}}\left(\mathbf{C}_{11}\right)$, in the range $40-45^{\circ}$, indicating carboxylic acid monolayers of high quality and surface coverage. On the contrary, the larger $\theta_{\mathrm{s}}$ of 70 and $68^{\circ}$ respectively for $1\left(\mathbf{C}_{11}\right)$ and $\mathbf{1}\left(\mathbf{C}_{16}\right)$ are a clear indication of incomplete disordered monolayers. Considering a reported correlation of surface density for binary homogeneous SAMs of undecanethiol and MUDA on $\mathrm{Au},{ }^{19}$ these values would correspond to a surface with only ca. $50 \%$ coverage by MUDA. Using the same correlation, $\theta_{\mathrm{s}}$ of $\mathbf{1}^{\mathrm{Au}}\left(\mathbf{C}_{11}\right), \mathbf{2}$ and $\mathbf{3}$ would correspond to a relative surface coverage $>90 \% .^{20}$

Monolayers were analysed by XPS, and characteristic survey and narrow scans are given in Figures S4-S9. Except for the Au-coated substrates where this is irrelevant, the absence of any significant amounts of silicon dioxide is confirmed by the flat baseline in the range 103-104 eV. Meanwhile, the presence of thiol molecules is confirmed by the detection of $S$ $2 p$ peaks. Deconvolution of the $C$ 1s narrow scans show the expected signal of oxidized carbons at ca. $288.5 \mathrm{eV}$ for the carboxylic terminated monolayers, in addition to the main peak of aliphatic carbons at $284.9 \mathrm{eV}$ and adventitious contaminations at ca. $286 \mathrm{eV}$. For the hydrosilylated monolayers, the silicon-bound carbons are also discernable at ca. 283.2-283.7 eV, and the relative ratios with the aliphatic carbons are close to those expected. Besides this chemical characterization, the analysis of the XPS data also evidences the differences in quality of the monolayers indicated by the contact angles, through much lower atomic $\mathrm{C} / \mathrm{Si}$ ratios in the case of thiol SAMs with respect to covalent monolayers obtained by hydrosilylation.

Table 1. Details of monolayers formed on oxide-free silicon together with their respective static contact angles $\theta_{\mathrm{s}}$, XPS atomic C/Si ratios and average surface roughness

\begin{tabular}{|c|c|c|c|c|c|}
\hline Code & Substrate & Method / reagent $^{\mathrm{a}}$ & $\begin{array}{l}\theta_{\mathrm{s}} \\
\left({ }^{\circ}\right)\end{array}$ & $\begin{array}{l}\text { atomic } \\
\text { ratio } \mathrm{C} / \mathrm{Si}\end{array}$ & $\begin{array}{l}\text { Roughness } \\
(\mathrm{nm})\end{array}$ \\
\hline $1\left(C_{11}\right)^{r e f}$ & $\mathrm{Si}(100)$ & $\mathrm{SAM}^{\mathrm{b}} / \mathrm{UDT}$ & 89 & $15.5 / 84.5$ & 0.24 \\
\hline $1\left(C_{11}\right)$ & $\mathrm{Si}(100)$ & $\mathrm{SAM}^{\mathrm{b}} / \mathrm{MUDA}$ & 70 & $19.1 / 80.9$ & 2.4 \\
\hline $1\left(C_{16}\right)$ & $\mathrm{Si}(100)$ & $\mathrm{SAM}^{\mathrm{b}} / \mathrm{HMDA}$ & 68 & $21.2 / 78.8$ & 1.4 \\
\hline $1^{\mathrm{Au}}\left(\mathrm{C}_{11}\right)^{\mathrm{ref}}$ & Au@Si & $\mathrm{SAM}^{\mathrm{b}} / \mathrm{UDT}$ & 90 & $\mathrm{~N} / \mathrm{A}$ & --- \\
\hline $1^{\mathrm{Au}}\left(\mathrm{C}_{11}\right)$ & Au@Si & $\mathrm{SAM}^{\mathrm{b}} / \mathrm{MUDA}$ & 40 & N/A & 0.2 \\
\hline $2^{\text {ref }}$ & $\mathrm{Si}(111)$ & $\begin{array}{l}\text { hydrosilylation }^{c} \text { / } \\
\text { undecene }\end{array}$ & 96 & $36.8 / 63.2$ & 1.3 \\
\hline 2 & $\mathrm{Si}(111)$ & $\begin{array}{l}\text { hydrosilylation }^{c} \\
\text { undecenoic acid }\end{array}$ & 45 & $33.2 / 66.8$ & 0.75 \\
\hline $3^{\text {ref }}$ & $\mathrm{Si}(111)$ & $\begin{array}{l}\text { hydrosilylation }^{\text {d }} \text { / } \\
\text { dodecyne }\end{array}$ & 98 & $36.9 / 63.1$ & --- \\
\hline 3 & $\mathrm{Si}(111)$ & $\begin{array}{l}\text { hydrosilylation }^{d} \\
\text { undecynoic acid }\end{array}$ & 42 & $32.8 / 67.2$ & 0.35 \\
\hline
\end{tabular}

${ }^{a}$ UDT = 1-undecanethiol, MUDA = 11-mercapto-undecanoic acid, HMDA = 16mercapto-hexadecanoic acid; ${ }^{b}$ thiol-based Self-Assembled Monolayers; thermally-induced; ${ }^{d}$ light-activated.

In fact, the atomic $\mathrm{C} / \mathrm{Si}$ ratio can be used to estimate the monolayer density and height. ${ }^{21,22}$ Here, the C/Si ratios for $2^{\text {ref }}$ and $3^{\text {ref }}$ are ca. 0.58 , similar to previously reported values for dense monolayers with a thickness of ca. $18 \AA^{23}$ The corresponding analysis done for the carboxylic acid terminated monolayers 2 and 3 give a slightly smaller value of ca. 0.49 , which would correspond to a monolayer thickness of ca. $16 \AA$, still pointing to well-ordered and dense monolayers. Comparatively, $\mathbf{1}\left(\mathbf{C}_{11}\right)$ and $\mathbf{1}\left(\mathbf{C}_{16}\right)$ have $\mathrm{C} / \mathrm{Si}$ ratio about 2.5 times smaller, i.e., the monolayers are about $8-10 \AA$ thin, confirming a very incomplete and disordered surface coverage. Atomic Force Microscopy (AFM) topographic images of $\mathbf{1}\left(\mathbf{C}_{11}\right), \mathbf{1}\left(\mathbf{C}_{16}\right)$ and $\mathbf{1}^{\mathrm{Au}}\left(\mathbf{C}_{11}\right)$ reveal that indeed the former two are less uniform, as indicated by their average roughness of 2.4 and $1.4 \mathrm{~nm}$, to be compared with a roughness of $0.6 \mathrm{~nm}$ for the latter, similar to the clean $\mathrm{Au}$ surface (Figures S16-S18). 

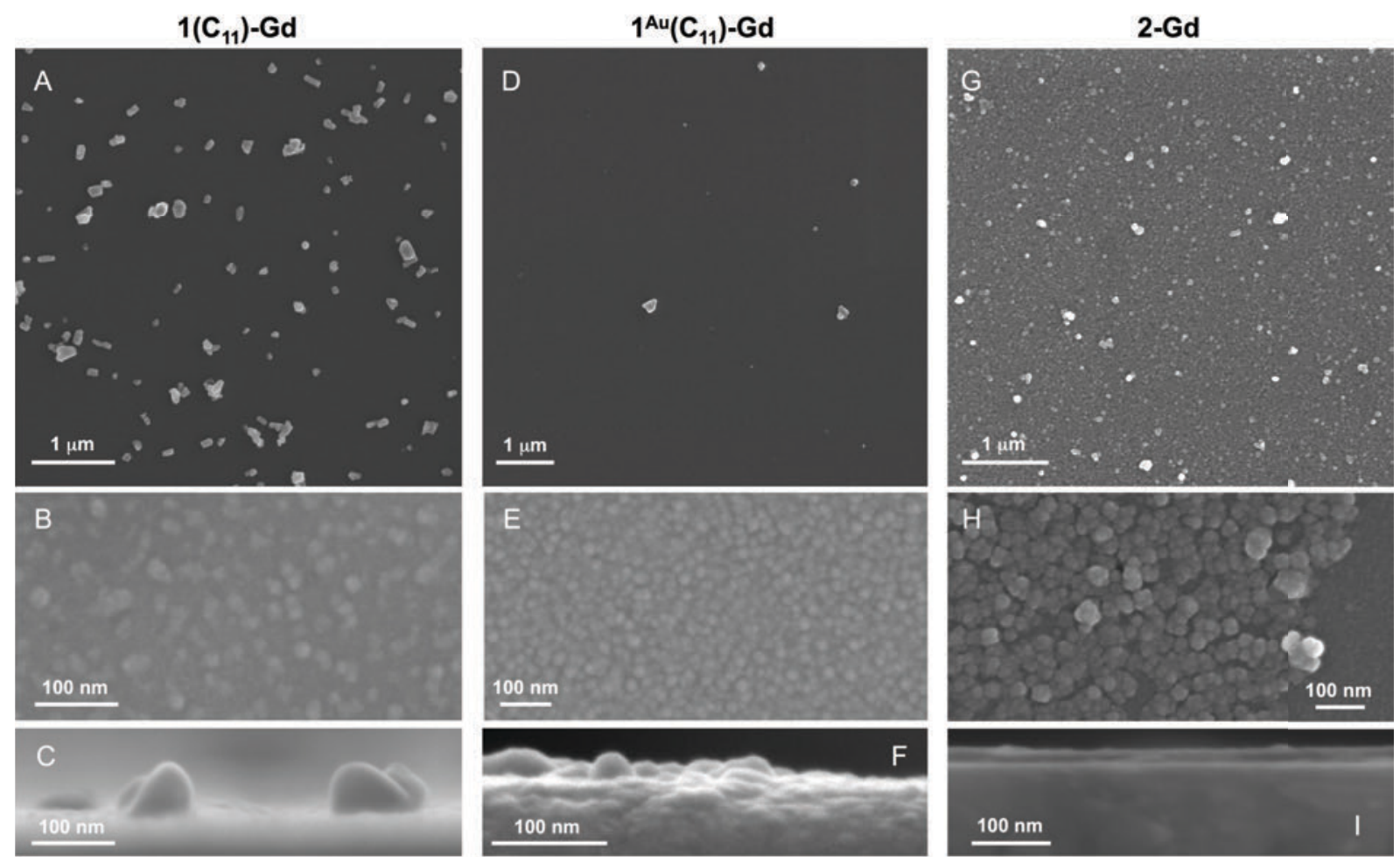

Fig. 1. Characteristic FESEM images of the $\mathrm{Gd}(\mathrm{HCOO})_{3}$ MOF grown on thiol-based SAM on oxide-free $\mathrm{Si}(100)\left(\mathbf{1}\left(\mathbf{C}_{\mathbf{1 1}}\right)\right.$ - $\mathbf{G d}$, left) and a Au layer grown on oxide-free $\mathrm{Si}(100)\left(\mathbf{1}^{\mathrm{Au}}\left(\mathbf{C}_{11}\right)-\mathbf{G d}\right.$, center), and covalent monolayer obtained by hydrosilylation of $\mathrm{Si}(111)(2-\mathrm{Gd}$, right). Images $\mathrm{C}, \mathrm{F}$ and $\mathrm{I}$ are cross-section views.

The lower roughness in the case of $\mathbf{1}\left(\mathrm{C}_{16}\right)$ is likely due to a relatively better order promoted by longer alkyl chains. The topography of all hydrosilylated monolayers is homogeneous with a roughness in the range $0.35-0.75 \mathrm{~nm}$, associated with the domains formed through this strategy. ${ }^{24}$ Interestingly, the height of the monolayer derived by mechanically scratching it from the $\mathrm{Si}(111)$ substrate is ca. $2.0 \mathrm{~nm}$, in good agreement with the estimation done on basis of the C/Si from XPS data.

Overall, monolayers with carboxylic acid end groups of various quality have been made on oxide-free Si. The thiol-based SAMs have a poor surface coverage and are likely disordered. While this can reasonably be associated with the much weaker Si-S interaction compared with the Au-S one, this does not necessarily impede the growth of the selected $\mathrm{Gd}(\mathrm{HCOO})_{3}$ MOF. Monolayers obtained by hydrosilylation on the contrary are dense and highly-ordered, and possess a further advantage in their high chemical and thermal stability, ${ }^{25}$ due to the formation of covalent $\mathrm{Si}-\mathrm{C}$ bonds. Alkynes normally form better (denser) monolayers than alkenes, ${ }^{23}$ but the relatively small difference may actually have no effect on MOF growth.

\subsection{Growth and characterization of gadolinium formate}

The same growth procedure was used for all freshly prepared carboxylic-terminated monolayers, involving immersion for 24 hours of the Si substrate in an ethanol solution of $\mathrm{Gd}(\mathrm{HCOO})_{3}$ close to saturation. The morphology of the formed material was analyzed by means of Field Emission Scanning Electron Microscopy (FESEM, Figures 1 and S11-S15) and AFM (Figure 2) measurements, revealing significant differences correlated with the quality of the subjacent monolayer.

For thin films grown on thiol-based SAMs $\mathbf{1}\left(\mathbf{C}_{11}\right)-\mathbf{G d}$ and $\mathbf{1}\left(\mathbf{C}_{16}\right)$-Gd, two different forms of grown material are present. Large nanocrystals with height of the order of 50-100 nm and length from 100 to $500 \mathrm{~nm}$ have formed but only cover a limited fraction of the whole surface (Figures $1 \mathrm{~A}$ and S11A), the remainder being covered by small to very small clusters, all this pointing to a 3D growth (Figures $1 \mathrm{~B}$ and S11B). Full coverage of the substrate surface is unclear from FESEM images. The root mean square (RMS) surface roughness is respectively $5.3 \mathrm{~nm}$ for $\mathbf{1}\left(\mathbf{C}_{11}\right)-\mathbf{G d}$ and of $2.1 \mathrm{~nm}$ for $\mathbf{1}\left(\mathbf{C}_{16}\right)$ - $\mathbf{G d}$, significantly higher than the bare SAMs and therefore pointing at a thin layer of grown material. This is also confirmed by the absence of these smaller objects when submitting the hydrophobic $1\left(C_{11}\right)^{\text {ref }}$ SAM to the same growth conditions (Fig. S12). The surface of $\mathbf{1}^{A u}\left(C_{11}\right)$-Gd almost only exhibits a similar thin layer of clusters, albeit more continuous and apparently thicker, plus only very few larger nanocrystals (Figure 1D-E). Eventually, the topography of $\mathbf{2 - G d}$ and $\mathbf{3 - G d}$ depicts a homogeneous complete coverage of the substrate by larger clusters of sizes in the range $15-40 \mathrm{~nm}$, and absence of large 

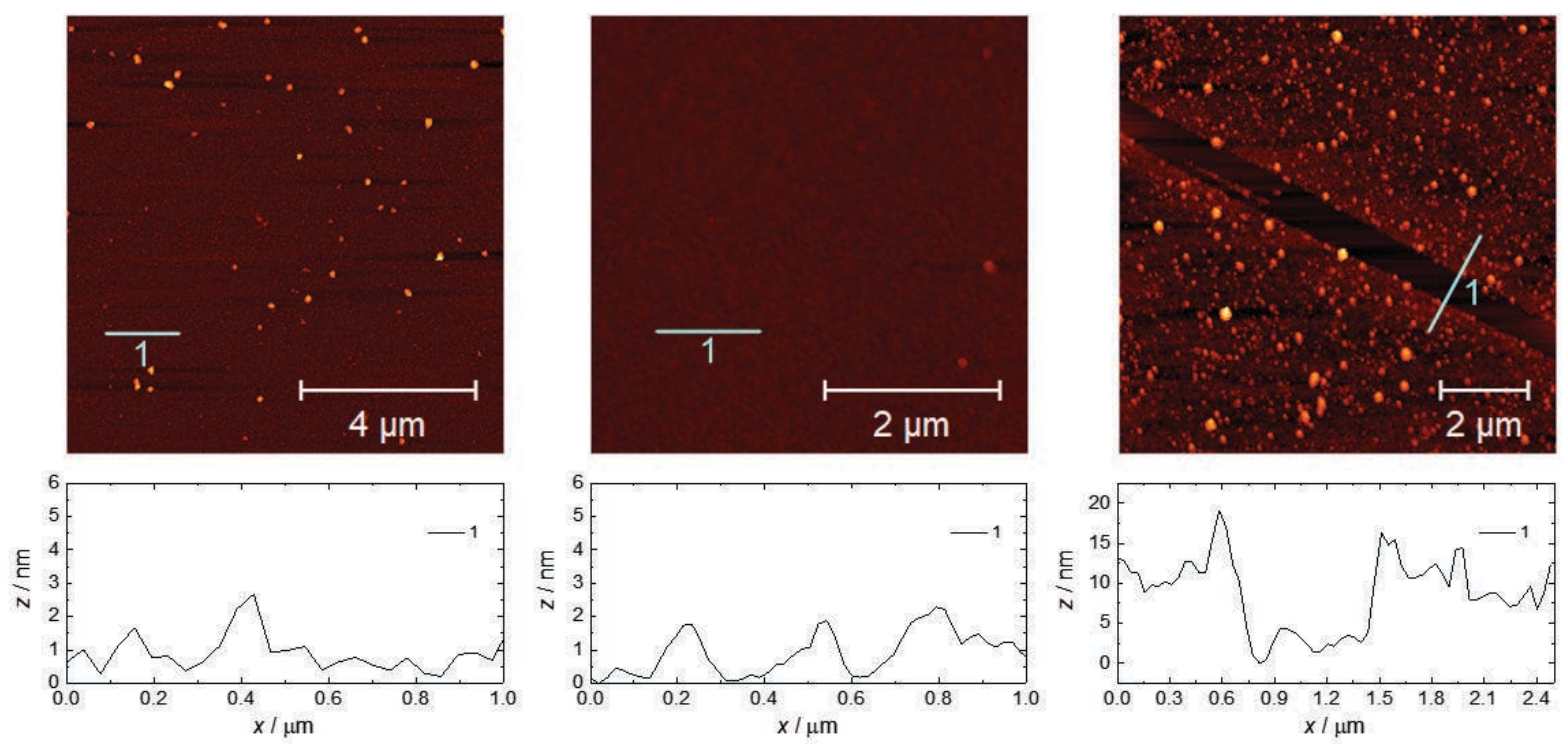

Fig. 2. AFM topography images and relative surface profiles for $\mathbf{1}\left(\mathbf{C}_{11}\right)-\mathbf{G d}$ (left), $\mathbf{1}^{\mathrm{Au}}\left(\mathbf{C}_{\mathbf{1 1}}\right)$-Gd (center) and $\mathbf{2}-\mathbf{G d}$ (right). The respective RMS surface roughness are $1.3,0.5$ and $4.3 \mathrm{~nm}$.

nanocrystals (Figure $1 \mathrm{G}-\mathrm{H}-\mathrm{I}$ and $\mathrm{S} 13)$. The rather homogenous size of the grown crystallites and complete coverage results in a still limited roughness of $0.5 \mathrm{~nm}$. The thickness of the grown film as evaluated through mechanical scratches remains limited though, at ca. $10 \mathrm{~nm}$. Nonetheless, the amount of the grown material can be increased by extending the immersion time of the functionalized substrate in the $\mathrm{Gd}(\mathrm{HCOO})_{3}$ solution (see below). Energy-dispersive X-ray spectroscopy (EDS) indicates the presence of $\mathrm{Gd}$ in the larger nano-crystals, but not in the cluster thin layers of $\mathbf{1}\left(\mathbf{C}_{11}\right)-\mathbf{G d}$, $\mathbf{1}\left(C_{16}\right)-G d$ and $1^{A u}\left(C_{11}\right)-G d$. This is however likely due to the limitations of EDS to detect elements in the most superficial part of a specimen. Anyhow, XPS confirms the presence of $\mathrm{Gd}$ in all thin films through the clear detection of $\mathrm{Gd} 3 d_{5 / 2}$ emission at $1185 \mathrm{eV}$ (Fig. S10). In addition, deconvolution of the $C$ 1s peaks indicates a significant relative increase of carboxylic acid $\mathrm{C}$, that can reasonably be associated with formate groups from $\mathrm{Gd}(\mathrm{HCOO})_{3}$. Therefore, the chemical composition of the grown crystallites agrees with the hypothesis that these are all the same $\mathrm{Gd}(\mathrm{HCOO})_{3}$ material. Eventually, X-ray diffraction confirms the identity of the material grown on both thiol and hydrosilylated monolayers (Figure 3). Patterns match those calculated from the singlecrystal structure of $\mathrm{Gd}(\mathrm{HCOO})_{3}$, demonstrating the successful growth of the selected MOF cooler on $\mathrm{Si}$ without impurity phases. Although the formed thin films are polycrystalline, the patterns indicate a significant preferential orientation along (110). An exception is $\mathbf{1}^{\mathrm{Au}}\left(\mathbf{C}_{11}\right)$-Gd for which the only (weak) diffraction peak corresponds to the (211) plane, pointing at a different growth mechanism for the continuous thin layer. This means that the diffraction patterns of $\mathbf{1}\left(\mathbf{C}_{11}\right)$-Gd and $\mathbf{1}\left(\mathbf{C}_{16}\right)-\mathbf{G d}$ are likely associated to the larger crystallites, in agreement with the relatively strong diffraction, detected with standard diffractometer. Obtaining diffraction patterns for $\mathbf{2 - G d}$ required grazing-incidence $X$-ray diffraction, likely due to the smaller size of the crystallites. The (110) preferred orientation observed for both $\mathbf{1}\left(\mathbf{C}_{11}\right)-\mathbf{G d}$ and $\mathbf{2 - G d}$ is one of the Gd-rich planes in the structure of $\mathrm{Gd}(\mathrm{HCOO})_{3}$ (Scheme 1), therefore corresponding to an optimal situation to initiate the growth through coordination of $\mathrm{Gd}(\mathrm{III})$ ions from the solution by the monolayer carboxylic end groups (Fig. 4).

Overall, $\mathrm{Gd}(\mathrm{HCOO})_{3}$ crystallites do grow on carboxylic acid monolayers on oxide-free $\mathrm{Si}$, albeit their size, morphology, and most importantly the growth efficiency clearly depend on the quality and type of the underlying monolayer. Even for the more favorable covalent monolayers obtained by hydrosilylation, the observed surface-growth of $\mathrm{Gd}(\mathrm{HCOO})_{3}$ is clearly less efficient than previously reported for a number of porous MOFs. ${ }^{14}$ These were however obtained by the socalled Liquid-Phase Epitaxy method, ${ }^{14,26}$ in which the substrate is exposed sequentially to the different components of the MOF, i.e., metal ions and linkers. We had originally attempted a similar method, using $\mathrm{GdCl}_{3}$ and ammonium formate solutions, but this proved unsuccessful. The sequential growth method is in principle optimal for MOFs with pillared layered structures, which could explain its inefficiency here with $\mathrm{Gd}(\mathrm{HCOO})_{3}$, although dense coatings have been successfully formed by this method with few other 3D unpillared MOFs. ${ }^{27}$ Another limitation is the solubility of $\mathrm{Gd}(\mathrm{HCOO})_{3}$ that does not allow to increase the concentration of the immersion solution, already close to saturation. Here, nevertheless, the probable most relevant parameter for the MOF growth is the metal ion itself and the associated lability and reactivity. Indeed, most carboxylate-based MOFs grown so far through sequential methods have been $\mathrm{Cu}$ (II) 
MOFs, ${ }^{14,26}$ many based on paddle-wheel $\mathrm{Cu}_{2}(\mathrm{RCOO})_{4}$ nodes, and therefore quite different from the connectivity in the structure of $\mathrm{Gd}(\mathrm{HCOO})_{3}$. Rapid ligand exchange kinetics typically occurring at $\mathrm{Cu}(\mathrm{II})$ ions are key for an efficient sequential layer-by-layer growth. $\mathrm{Gd}(\mathrm{III})$ is comparatively a kinetically inert ion and this could explain a comparatively poor on-surface growth.

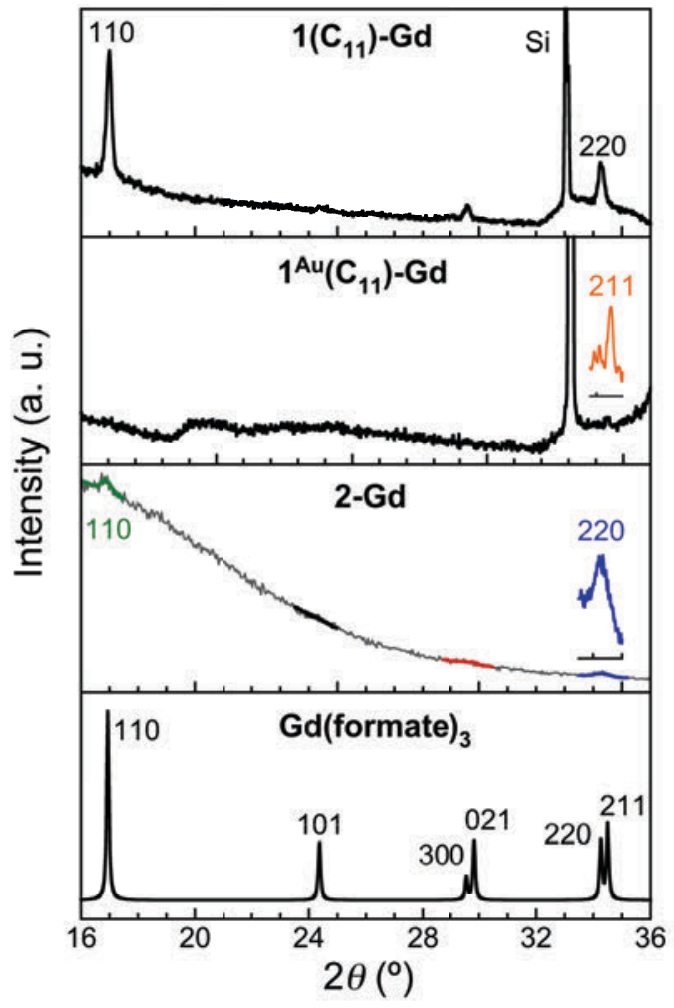

Fig. 3. X-ray diffraction patterns for $\mathbf{1}\left(\mathrm{C}_{11}\right)-\mathbf{G d}, \mathbf{1}^{\mathrm{Au}}\left(\mathrm{C}_{\mathbf{1 1}}\right)$-Gd and $\mathbf{2}-\mathbf{G d}$, compared with the calculated patterns from the single-crystal structure of $\mathrm{Gd}(\mathrm{HCOO})_{3}$, as indicated. The practical absence of the (101), (300) and (021) peaks in the patterns of $\mathbf{1}\left(\mathbf{C}_{11}\right)$-Gd and $\mathbf{2}-\mathbf{G d}$ point at a preferential orientation along 110 .

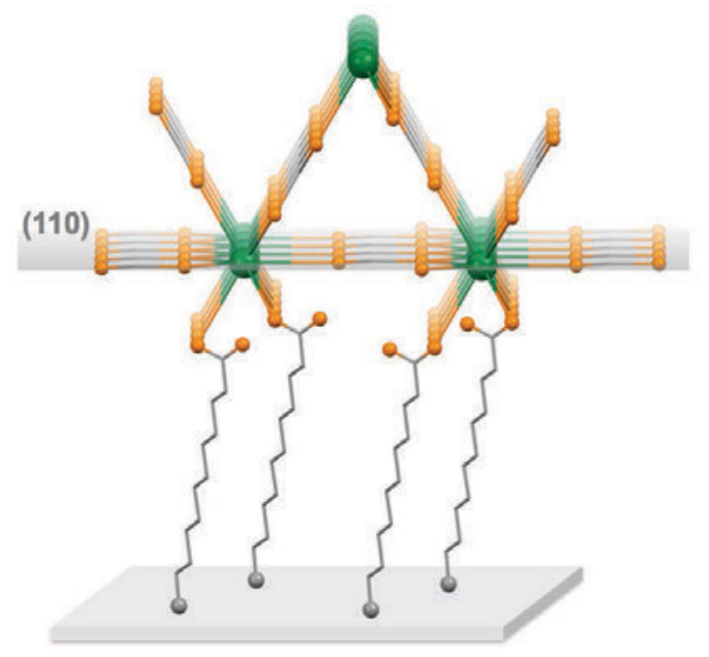

Fig. 4. Representation of the main preferred $\mathrm{Gd}(\mathrm{HCOO})_{3}$ growth direction on the carboxylate terminated monolayers. The coordination anchoring mode is hypothetic. Colour code: Gd, green spheres; O, orange spheres; C, grey sticks. The grey spheres represent the connection to the silicon, either through a S-Si interaction or a C-Si covalent bond.
Besides the simple 24 hours immersion, we have also tested multiple shorter immersions, which lead to no significant modifications in terms of quality, homogeneity and quantity of grown material. In particular, repeated short immersions of $\mathbf{1}\left(\mathbf{C}_{11}\right)$ does not impede the growth of large crystallites. These are also formed when using a 10 -fold diluted $\mathrm{Gd}(\mathrm{HCOO})_{3}$ solution (Figure S14). Altogether, this would indicate that the formation of these large crystallites is associated with the comparatively poorer quality of the thiolated SAM, that likely results in preferential nucleation and growth at either defects or, more likely, well-ordered areas.

Longer immersions of 48 hours were also performed on 2 , both refreshing the $\mathrm{Gd}(\mathrm{HCOO})_{3}$ solution after 24 hours or using the same solution for the whole 48 hours $\left(2-G^{48 h}\right)$. FESEM observations (Figure S15) together with estimation of the amount of material grown through magnetic measurements (see next section) indicate that the amount of surface-grown $\mathrm{Gd}(\mathrm{HCOO})_{3}$ can be increased through the immersion time. Such ability to adjust the amount of grown material is particularly important for the proper cooling of the substrate, as discussed in next Section. The only downside could be a comparatively less homogeneous thin film, as indicated by the observation for $\mathbf{2}-\mathbf{G d}^{\mathbf{4 8 h}}$ of some larger crystallites on top of a continuous film similar to that observed for 2-Gd.

Table 2. Average RMS roughness, XPS atomic $\mathrm{Gd} / \mathrm{Si}$ ratios and surface density for the $\mathrm{Gd}(\mathrm{HCOO})_{3}$ thin films grown on oxide-free silicon with different subjacent carboxylic monolayers.

\begin{tabular}{|c|c|c|c|}
\hline sample & roughness & atomic ratio $\mathrm{Gd} / \mathrm{Si}$ & $\begin{array}{l}\text { surface density } \\
{\left[10^{-7} \mathrm{~g} / \mathrm{cm}^{2}\right]}\end{array}$ \\
\hline $1\left(C_{11}\right)-G d$ & 5.3 & 0.00035 & 2.4 \\
\hline $1\left(C_{16}\right)-G d$ & 2.1 & 0.00041 & 2.9 \\
\hline $1^{A u}\left(C_{11}\right)-G d$ & 0.75 & $\mathrm{~N} / \mathrm{A}$ & 2.9 \\
\hline $2-G d$ & 4.7 & 0.00067 & 20.2 \\
\hline $2-G d^{48 h}$ & $2.75^{\mathrm{a}}$ & - & 43.8 \\
\hline 3-Gd & 0.4 & 0.00131 & 28.6 \\
\hline
\end{tabular}

${ }^{a}$ for $\overline{\mathbf{2 - G d}}{ }^{48}$, few bunches of large crystallites are present on top of the main deposit. The roughness given is for areas without such larger crystallites and therefore describing the grown material. Roughness of large areas (e.g., 10 $\mathrm{m} \mathrm{x}$ $10 \mu \mathrm{m}$ or larger) containing some larger crystallites is $>21$.

\subsection{Magnetic properties and refrigeration potential.}

The magnetization of the made thin films was found sufficient to be detected at low temperatures by standard RSO-SQUID magnetometry $\left(\sim 10^{-8}-10^{-9} \mathrm{emu}\right)$, thus allowing a measure of the amount of $\mathrm{Gd}(\mathrm{HCOO})_{3}$ grown on each type of substrate (see supporting information for details, Figures S19-S21). The temperature dependence of the magnetic susceptibility in the range $2-50 \mathrm{~K}$ and isothermal magnetization at 2 and $5 \mathrm{~K}$ were determined for the $\mathrm{Gd}(\mathrm{HCOO})_{3}$ grown films in $\mathbf{1}\left(\mathbf{C}_{11}\right)-\mathbf{G d}$, 1(C $\left.C_{16}\right)-G d, 1^{A u}\left(C_{11}\right)-G d, 2-G d, 3-G d$ and $2-G^{48 h}$ (Figures 5 


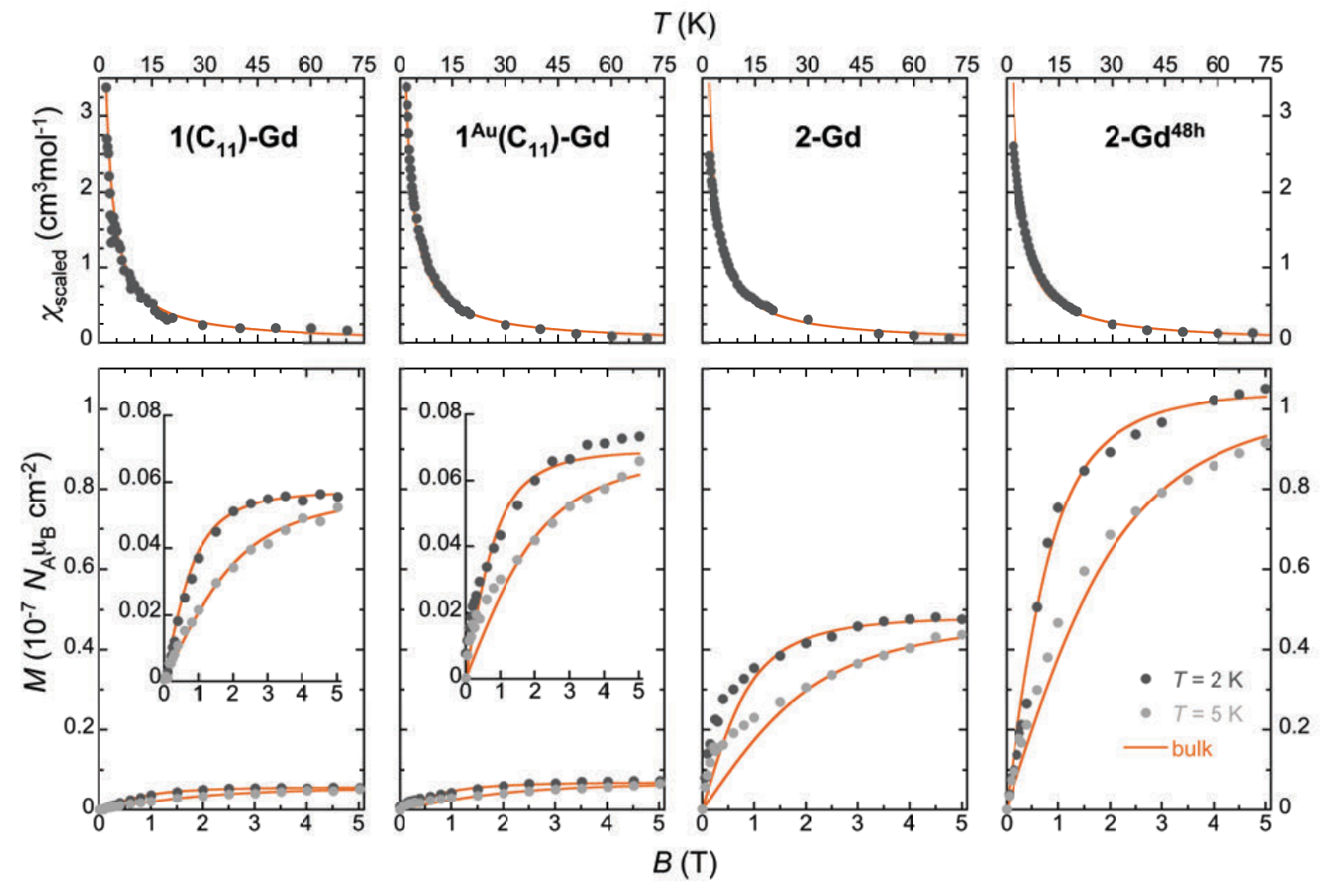

Fig. 5. Magnetic properties of the $\mathrm{Gd}(\mathrm{HCOO})_{3}$ thin films grown on oxide-free silicon with different subjacent carboxylic monolayers as indicated. Top: temperature dependence of the molar magnetic susceptibility obtained by scaling to the bulk material data shown as orange lines. Bottom: isothermal field dependence of the molar magnetization per surface area at 2 and $5 \mathrm{~K}$, as determined by comparison with the bulk material data (see supporting information for details). The data is shown using the same range to evidence the significant differences arising from the subjacent carboxylate monolayers and growth time. For $\mathbf{1}\left(\mathbf{C}_{11}\right)$-Gd and $\mathbf{1}^{\mathrm{Au}}\left(\mathbf{C}_{\mathbf{1 1}}\right)$ Gd, the data is also shown with a more adequate range as insets. The scaled bulk material data is shown as orange lines.

and S22). For this, the diamagnetic contribution of the bare 100 and $111 \mathrm{Si}$ wafers were experimentally determined under the same isofield and isothermal conditions and subtracted to the corresponding total magnetization, thus providing the raw magnetic response of the grown materials (Figure S19). Given that the amount of deposited material is initially unknown, these data, obtained in emu units, are then scaled to the molar magnetization and susceptibilities of the bulk $\mathrm{Gd}(\mathrm{HCOO})_{3}$ parent material (solid lines in Fig. 4, Figure S20). For all the samples, the agreement is good for both magnetization and susceptibility experiments, thus demonstrating that the magnetic properties of $\mathrm{Gd}(\mathrm{HCOO})_{3}$, and so its corresponding MCE, are maintained in the surface-grown nano-crystals. Noteworthy, the same factor used to scale the $M$ vs. $B$ data collected for 2 and $5 \mathrm{~K}$ can also be employed for scaling satisfactorily the $M$ vs. $T$ data for each sample. As the scaling procedure involves separate experiments, this ensures the reliability of the results and, indirectly, an estimate of the mass of the grown materials. The obtained $\mathrm{Gd}(\mathrm{HCOO})_{3}$ surface densities indicate significant differences between growth on thiol-based SAMs and hydrosylilated covalent monolayers, in line with the semi-quantitative $\mathrm{Gd} / \mathrm{C}$ ratios determined by XPS (Table 2). Indeed, while the growth conditions and time are identical, 2-Gd and 3-Gd have $\mathrm{Gd}(\mathrm{HCOO})_{3}$ surface densities about one order of magnitude larger than those of $1\left(C_{11}\right)-G d$, $\mathbf{1}\left(C_{16}\right)-G d$ and $1^{A u}\left(C_{11}\right)-G d$. The similar surface densities for the latter three can be reasoned in light of the presence or absence thereof of large crystals. Indeed, while the better quality of the subjacent SAM for $\mathbf{1}^{\mathrm{Au}}\left(\mathbf{C}_{11}\right)$-Gd gives rise to a more homogeneous layer than for $\mathbf{1}\left(\mathbf{C}_{11}\right)$-Gd and $\mathbf{1}\left(\mathbf{C}_{16}\right)$-Gd, this is likely compensated by the volume of the larger crystals present only in the latter two samples. Then, the 10 -fold increase of surface density between $\mathbf{1}^{\mathrm{Au}}\left(\mathbf{C}_{11}\right)$-Gd and $\mathbf{2}-\mathbf{G d}$ and 3-Gd, all with dense ordered subjacent carboxylic acid monolayers, necessarily means that not only its quality but also its type, i.e., physisorbed vs. covalent, is relevant to the MOF growth, as already indicated by FESEM observations. Here, it is significant that the growth occurs in $\mathrm{EtOH}$, the same solvent used to elaborate the thiol-based SAMs. The dynamic processes occurring for thiol-based SAMs in contact with their solution are therefore active, and may represent a limitation for a MOF growth involving coordination bond formation with kinetically inert $\mathrm{Gd}(\mathrm{III})$ ions.

Next, we evaluate the cooling potential of our $\mathrm{Gd}(\mathrm{HCOO})_{3}$ thin films. In an on-chip microrefrigerator, the magnetocaloric 
material would be deposited on a silicon substrate, ${ }^{12}$ manufactured in the form of a suspended membrane (see Scheme 1). Heat transfer has to proceed via low-energy phonons, because of the low working temperatures. The anchor molecules with a carboxylic end function should not hinder the conduction of heat between the $\mathrm{Gd}(\mathrm{HCOO})_{3}$ crystallites and the silicon substrate. The soft, flexible nature of the aliphatic chains of the anchor molecules provides numerous degrees of freedom and, therefore, they should couple well with low-temperature vibrational modes.

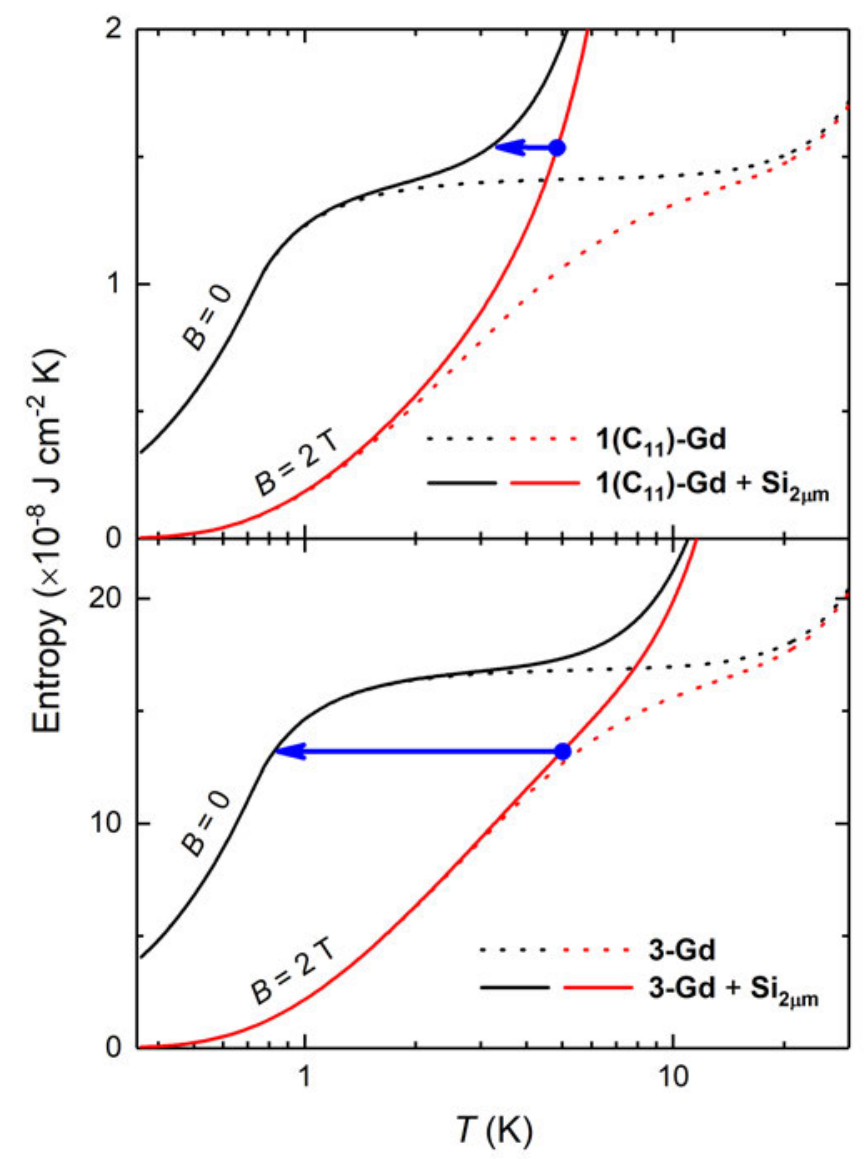

Fig. 6. Temperature dependence of the entropy for $B=0$ and $2 \mathrm{~T}$, normalized to the surface area of the substrate, for $\mathbf{1}\left(\mathbf{C}_{11}\right)-\mathbf{G d}$ (top) and $\mathbf{3 - G d}$ (bottom), with (solid lines) and without (dotted lines) the contribution of a thermally-contacted $\mathrm{Si}$ membrane of $2 \mu \mathrm{m}$ thick $\left(\mathrm{Si}_{2 \mathrm{~m}}\right)$, as indicated. The blue arrow indicates an adiabatic demagnetization of the MOF material that, starting from $T=5 \mathrm{~K}$ and $B$ $=2 \mathrm{~T}$, lowers its temperature and that of $\mathrm{Si}_{2 . \mathrm{m}}$ down to $0.8 \mathrm{~K}$ for $\mathbf{3}-\mathbf{G d}$ and, only, $3.3 \mathrm{~K}$ for $\mathbf{1}\left(\mathbf{C}_{11}\right)-\mathbf{G d}$, when reaching zero applied field.

We tentatively assume that the membrane has a thickness of $2 \mu \mathrm{m}$ and that its low-temperature heat capacity is significantly larger than that of any other component of the device but the $\mathrm{Gd}(\mathrm{HCOO})_{3}$ thin film. We also assume ideal experimental conditions, that is, the refrigeration by demagnetization proceeds adiabatically. For the sake of exemplification, let us focus on the representative $\mathbf{1}\left(\mathbf{C}_{11}\right)-\mathbf{G d}$ and $\mathbf{3 - G d}$ solely, and on starting conditions of the adiabatic demagnetization that can be easily accessed by cryocoolers and permanent magnets, e.g., $T=5 \mathrm{~K}$ and $B=2 \mathrm{~T}$, respectively. Figure 6 shows the temperature dependencies of the entropies for $\mathbf{1}\left(\mathbf{C}_{11}\right)-\mathbf{G d}$ and
3-Gd alone (dotted lines), together with the entropies of the system (solid lines) that comprises either $\mathbf{1}\left(\mathbf{C}_{11}\right)$ - $\mathbf{G d}$ or $\mathbf{3 - G d}$ and the Si membrane of $2 \mathrm{~mm}$ thick $\left(\mathrm{Si}_{2 . \mathrm{m}}\right)$, thermally linked to the corresponding MOF material. The contributions of $\mathbf{1}\left(\mathrm{C}_{11}\right)$ Gd and 3-Gd are obtained from the experimentally determined entropy of the parental $\mathrm{Gd}(\mathrm{HCOO})_{3}$ bulk material, ${ }^{8 a}$ properly rescaled by the corresponding surface densities (Table 2). The contribution of $\mathrm{Si}_{2, \mathrm{~m}}$ is calculated from the Debye temperature of $645 \mathrm{~K}$, as for silicon, which is then added to the entropies of $\mathbf{1}\left(\mathbf{C}_{11}\right)$-Gd and $\mathbf{3 - G d}$, respectively (Fig. 6). The lowest temperature that can be attained by the proposed adiabatic demagnetization (blue arrows in Fig. 6) is markedly different, depending on the representative film considered. Specifically, 3-Gd permits reaching ca. $0.8 \mathrm{~K}$ after a full demagnetization down to zero applied field, whilst only ca. 3.3 $\mathrm{K}$ can be attained with $\mathbf{1}\left(\mathbf{C}_{11}\right)-\mathbf{G d}$ under the same conditions, making the latter magnetocaloric thin film barely useful for the proposed target. The reason of such a difference is nothing but the relative amount of magnetocaloric material present, viz., the higher its surface density, the relatively lower is the contribution of $\mathrm{Si}_{2, m}$ and, therefore, the resulting temperature change is closer to bulk behavior. Interestingly, the surface density of grown $\mathrm{Gd}(\mathrm{HCOO})_{3}$ is found to double when growth time on hydrosylilated covalent monolayers is increased from 24 to 48 hours. This clearly demonstrates that thicker films of $\mathrm{Gd}(\mathrm{HCOO})_{3}$ can be grown, to provide the necessary and sufficiently large refrigeration capacity. Therefore Si membranes, that are now developed and used for a number of applications, ${ }^{28}$ would be efficiently cooled by our still unoptimized gadolinium formate thin layers.

\section{Conclusions}

The gadolinium formate MOF can be grown on oxide-free $\mathrm{Si}$ covered with carboxylate-terminated monolayers by immersion in a concentrated solution of the bulk material at ambient temperature. This provides a functionalized surface with a local source of magnetic refrigeration for temperatures below $10 \mathrm{~K}$, through the large magneto-caloric effect of gadolinium formate. The quality and type of the carboxylateterminated monolayer is however critical for the growth efficiency and therefore the amount of grown MOF and its associated cooling power. Covalent monolayers obtained by thermal or photo-induced hydrosilylation of $\mathrm{Si}(111)-\mathrm{H}$ surfaces give the best result, likely due to their high quality and full surface coverage, as well as their robustness compared to physisorbed SAMs. The hydrosilylation strategy also has the advantage of being applicable to oxide-free $\mathrm{Si}_{3} \mathrm{~N}_{4},{ }^{29}$ the other material with a low-temperature small heat capacity and used as device support. Importantly, the amount of grown MOF can be increased accordingly to the immersion time. The still rather thin MOF layers reported here already provide an estimated relative cooling power per surface area sufficient to efficiently cool $\mathrm{Si}$ or $\mathrm{Si}_{3} \mathrm{~N}_{4}$ membranes currently developed for a number of applications. This work is the first exploration of growth of a $\mathrm{Gd}(\mathrm{III}) \mathrm{MOF}$ on oxide-free $\mathrm{Si}$, and improvements may be expected from considering other carboxylate-based MOFs for which the method used here should be extendable. 
Increasing the growth temperature may also be useful to improve the quality and thickness of Gd(III) MOF layers, as it is already known to strongly influence on-surface growth of MOFs with $\mathrm{Cu}(\mathrm{II}),{ }^{30}$ a highly labile metal ion compared to $\mathrm{Gd}(\mathrm{III})$. Altogether, our results show that local on-chip magnetic refrigeration at cryogenic temperatures may be provided by thin layers of molecular-based coolers, and we are currently working on directly measure their cooling efficiency.

\section{Conflicts of interest}

There are no conflicts of interest to declare.

\section{Acknowledgements}

Authors acknowledge funding from the Spanish MINECO through projects MAT2014-53961-R (GL, EN, OR), MAT201570868-ERC (OR), MAT2015-68204-R (GL, ME) and MAT2017-86826-R (OR, EN), as well as from the Aragón government (DGA, consolidated groups MOLCHIP E98, PLATON E31_17R and M4 E13_17R). The authors are grateful to Dr. G. Antorrena (LMA) for his help with XPS and GIXRD experiments, to Dr. J. Sesé for providing the Si wafers and to Dr. J. Stankiewicz for her help with the Au deposition.

\section{References}

1 a) M. N. Leuenberger and D. Loss, Nature 2001, 410, 789; b) Molecular Magnets: Physics and Applications, eds. J. Bartolomé, F. Luis and J. F. Fernández, Springer, 2014

2 a) A. Ardavan, O. Rival, J. Morton, S. Blundell, A. Tyryshkin, G. Timco and R. E. P. Winpenny, Phys. Rev. Lett. 2007, 98, 057201; b) G. Aromí, D. Aguilà, P. Gamez, F. Luis and O. Roubeau, Chem. Soc. Rev. 2012, 41, 537

${ }^{3}$ a) M. Evangelisti and E. K. Brechin, Dalton Trans. 2010, 39, 4672; b) R. Sessoli, Angew. Chem. Int. Ed. 2012, 51, 43

${ }^{4}$ a) W. F. Giauque, J. Am. Chem. Soc. 1927, 49, 1864; b) R. P. Hudson, Principles and Applications of Magnetic Cooling, North-Holland, Amsterdam 1972; c) V. K. Pecharsky and K. A. Gschneider, J. Magn. Magn. Mater. 1999, 25, 44

${ }^{5}$ a) A. M. Tishin, Y. I. Spichkin, The magnetocaloric effect and its applications (Series in Condensed Matter Physics), IOP, Bristol 2003; b) V. Franco, J. S. Blázquez, J. J. Ipus, J. Y. Law, L. M. Moreno-Ramírez, A. Conde, Prog. Mater. Sci. 2018, 93, 112

${ }^{6}$ a) A. T. Serlemitsos, B. A. Warner, S. Castles and S. R. Breon, Adv. Cryogenics 1990, 35B, 1431; b) P. J. Shirron, Cryogenics 2014, 62, 130

${ }^{7}$ examples of molecular coolers with high volumetric MCE: a) M. Evangelisti, O. Roubeau, E. Palacios, A. Camón, T. N. Hooper, E. K. Brechin and J. J. Alonso, Angew. Chem. Int. Ed. 2011, 50, 6606; b) L.-X. Chang, G. Xiong, L. Wang, P. Cheng and B. Zhao, Chem. Commun. 2013, 49, 1055; c) F.-S- Guo, Y.-C. Chen, L.-L. Mao, W.-Q. Li, J.-D. Leng, R. Tarasenko, M. Orendác, J. Prokleska, V. Schovsky and M.-L. Tong, Chem. Eur. J. 2013, 19, 14876
${ }^{8}$ examples of Metal-Organic Frameworks with high volumetric MCE: a) G. Lorusso, J. W. Sharples, E. Palacios, O. Roubeau, E. K. Brechin, R. Sessoli, A. Rossin, F. Tuna, E. J. L. Mclnnes, D. Collison and M. Evangelisti, Adv. Mater. 2013, 25, 4653; b) R. Sibille, T. Mazet, B. Malaman and M. François, Chem. Eur. J. 2012, 18, 12970; c) G. Lorusso, M. A. Palacios, G. S. Nichol, E. K. Brechin, O. Roubeau and M. Evangelisti, Chem. Commun. 2012, 48, 7592; d) Y-C. Chen, L. Qin, Z-S. Meng, D-F. Yang, C. Wu, Z. Fu, Y-Z. Zheng, J-L. Liu, R. Tarasenko, M. Orendác, J. Prokleska, V. Sechosky and M-L. Tong, J. Mater. Chem. A 2014, 2, 9851; e) C. Das, A. Upadhyay, K. Uddin Ansari, N. Ogiwara, T. Kitao, S. Horike, M. Shanmugam, Inorg. Chem. 2018, 57, 6584

9 a) J. W. Sharples and D. Collison, Polyhedron 2013, 54, 91; b) M. Evangelisti, "Molecule-based magnetic coolers: measurement, design and application", in Molecular Magnets. Physics and Applications, ed. F. Luis, J. Bartolomé and J. F. Fernandez, Springer-Verlag, Berlin, Heidelberg, 2014, pp. 365-387

${ }^{10}$ O. Roubeau, E. Natividad, M. Evangelisti, G. Lorusso and E. Palacios, Mater. Horiz. 2017, 4, 464

${ }^{11}$ V. Corradini, A. Ghirri, A. Candini, R. Biagi, U. del Pennino, G. Dotti, E. Otero, F. Choueikani, R. J. Blagg, E. J. L. Mclnnes and M. Affronte, Adv. Mater. 2013, 25, 2816

12 G. Lorusso, M. Jenkins, P. Gónzalez-Monje, A. Arauzo, J. Sesé, D. Ruiz-Molina, O. Roubeau and M. Evangelisti, Adv. Mater. 2013, 25, 2984

${ }^{13}$ Electronic schemes currently exploited produce only limited DT of the order of $0.1 \mathrm{~K}$, see J. T. Muhonen, M. Meschke and J. P. Pekola, Rep. Prog. Phys. 2012, 75, 046501

14 a) A. Bétard and R. A. Fischer, Chem. Rev. 2012, 112, 1055; b) J. Liu and C. Wöll, Chem. Soc. Rev. 2017, 46, 5730

15 this has been assessed due its importance in Si-based electronics, see a) O. W. Käding, H. Skurk. And K. E. Goodson, Appl. Phys. Lett. 1994, 65, 1621; b) L. J. McDaid, S. Hall, P. H. Mellor, W. Eccleston and J. C. Alderman, Electron. Lett. 1989, 25, 827

${ }^{16}$ a) M. R. Linford, P. Fenter, P. M. Eisenberger and C. E. D. Chidsey, J. Am. Chem. Soc. 1995, 117, 3145; b) A. B. Sieval, A. L. Demirel, J. W. M. Nissink, M. R. Linford, J. H. Van der Maas, W. H. De Jeu, H. Zuilhof and E. J. R. Sudhölter, Langmuir 1998, 14, 1759; c ) P. Thissen, O. Seitz and Y. J. Chabal, Prog. Surf. Sci. 2012, 87, 272

17 L. Scheres, A. Arafat and H. Zuilhof, Langmuir 2007, 23, 8343

${ }^{18}$ reported values under optimized conditions are respectively $100^{\circ}$ and $108^{\circ}$. The larger difference obtained here with dodecyne can be ascribed to the shorter alkyl chain, as this has been shown to affect the surface coverage when using alkynes while having no effect when using alkenes, see ref. 23.

19 S-I. Imabayashi, N. Gon, T. Sasaki, D. Hobara and T. Takiuchi, Langmuir 1998, 14, 2348

20 note that for the hydrosilylated substrates, this is not corresponding to the true surface coverage, i.e. the fraction of surface atoms that have formed a $\mathrm{Si}-\mathrm{C}$ bond. It has been 
shown that surface coverages of up to 55 and $65 \%$ can be reached respectively with alkenes and alkynes, see ref. 23 .

${ }^{21} \mathrm{X}$. Wallart, C. Henry de Villeneuve and P. Allongue, J. Am. Chem. Soc. 2005, 127, 7871

${ }^{22}$ using the relation $d_{\mathrm{ML}}(\AA)=\Lambda^{\mathrm{Si}}{ }_{\mathrm{ML}} \sin (j) \ln (1+\mathrm{C} / \mathrm{Si})$, where $\Gamma^{\mathrm{Si}}{ }_{\mathrm{ML}}$ is the attenuation length of $\mathrm{Si}_{2 p}$ photoelectrons in the organic monolayer and $j$ is the angle between the surface plane and the detector, taken as $90^{\circ}$; see ref. 21 . The derived thicknesses should however be taken only as rough estimations because the $\mathrm{C} / \mathrm{Si}$ ratios obtained here are not truly quantitative. This is because the influence of photodiffraction on the XPS signal is not taken into account as done in ref. 21 by rotating the samples by $360^{\circ}$ around the surface normal to yield rotationally averaged $C$ s and Si $2 p$ emissions.

23 a) L. Scheres, M. Giesbers and H. Zuilhof, Langmuir 2010, 26, 4790-4795; b) L. Scheres, M. Giesbers and H. Zuilhof, Langmuir 2010, 26, 10924

24 a) B. J. Eves, Q-Y. Sun, G. P. Lopinski and H. Zuilhof, J. Am. Chem. Soc. 2004, 126, 14318; b) B. J. Eves and G. P. Lopinski, Langmuir 2006, 22, 3180

${ }^{25}$ N. S. Bhairamagdi, S. P. Pujari, F. G. Trovela, A. Debrassi, A. A. Kahmos, J. M. Alonso, A. A. Al Zahrani, T. Wenneles, H: A. Al-Turaif, C. Van Rijn, Y. A. Alhamed and H. Zuilhof, Langmuir 2014, 30, 5829

26 J-L. Zhuang, A. Terfort and C. Wöll, Coord. Chem. Rev. 2016, 307, 391

${ }^{27}$ V. Stavila, C. Schneider, C. Mowry, T. R. Zeitler, J. A. Greathouse, A. L. Robinson, J. M. Denning, J. Volponi, K. Leong, W. Quan, M. Tu, R. A. Fischer and M. D. Allendorf, Adv. Funct. Mater. 2016, 26, 1699

28 Silicon nanomembranes. Fundamental Science and applications, eds. J. A. Rogers, J-H. Ahn, Wiley-VCH, Weinheim, 2016

${ }^{29}$ A. Arafat, M. Giesbers, M. Rosso, E. J. R. Sudhölter, K. Schroën, R. G. White, L. Yang, M. R. Linford and H. Zuilhof, Langmuir 2007, 23, 6233

${ }^{30} \mathrm{~J}$-L. Zhuang, M. Kind, C. M. Grytz, F. Farr, M. Diefenbach, S. Tussupbayev, M. C. Holthausen and A. Terfort, J. Am. Chem. Soc. 2015, 137, 8237 